\title{
Enhanced cerebral bioenergetics with dietary ketosis in Mild Cognitive Impairment
}

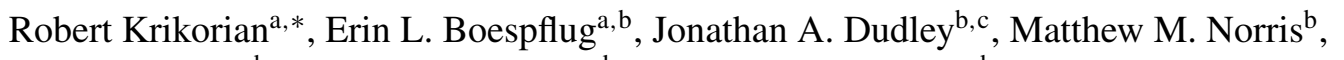 \\ Wen-Jang Chu ${ }^{\mathrm{a}, \mathrm{b}}$, Suzanne S. Summer ${ }^{\mathrm{d}}$ and James C. Eliassen ${ }^{\mathrm{a}, \mathrm{b}}$ \\ ${ }^{a}$ Department of Psychiatry \& Behavioral Neuroscience, University of Cincinnati, Cincinnati, OH, USA \\ ${ }^{\mathrm{b}}$ Center for Imaging Research, University of Cincinnati, Cincinnati, OH, USA \\ ${ }^{c}$ Department of Biomedical Engineering, University of Cincinnati, Cincinnati, OH, USA \\ ${ }^{\mathrm{d}}$ Clinical Translational Research Center, Cincinnati Children's Hospital Medical Center, Cincinnati, OH, USA
}

\begin{abstract}
.
BACKGROUND: Metabolic disturbance is associated with risk for neurodegeneration, and cerebral glucose hypometabolism is prominent in Alzheimer's disease (AD). Ketone metabolism can compensate for glucose hypometabolism and confers other benefits pertinent to neurodegeneration, among them reduction of oxidative stress and AD pathological factors, suggesting its potential as a therapeutic intervention. In a prior controlled trial, we showed that six weeks' carbohydrate restriction induced ketogenesis and produced improvements in metabolic parameters and memory performance in older adults with Mild Cognitive Impairment (MCI). Those benefits were attributed to the correction of hyperinsulinemia and to the presumed enhancement of cerebral bioenergetic function associated with ketone metabolism.

OBJECTIVE: To assess the effect of dietary ketosis on cerebral metabolites in older adults with MCI.

METHODS: We enrolled a sample of five MCI participants in a ketogenic dietary regimen and performed pre- and postintervention proton magnetic resonance spectroscopy to investigate changes in neurochemical metabolites. We also assessed cognitive function and metabolic and anthropometric factors.

RESULTS: We observed a significant increase in myo-inositol $(p=0.02)$ and trends for increases in N-acetyl-aspartate $(p=0.09)$ and creatine + phosphocreatine $(p=0.11)$ after six weeks on the ketogenic regimen. Working memory $(p=0.01)$ and long-term memory $(p=0.07)$ performances also improved.

CONCLUSIONS: This study offers novel, preliminary evidence of cerebral bioenergetic enhancement with mild dietary ketosis in aging humans. Further investigation in controlled trials is warranted to assess the preventive and treatment implications of this intervention for age-related memory decline and dementia.
\end{abstract}

Keywords: Ketones, proton MRS, glucose hypometabolism, memory, Mild Cognitive Impairment, Alzheimer's disease

\section{Introduction}

Insulin resistance syndrome is a common condition among older adults, affecting more than $40 \%$ of individuals at age 60, with increasing prevalence with aging [1]. Older adults with insulin resis-

${ }^{*}$ Corresponding author: Robert Krikorian, PhD, Department of Psychiatry \& Behavioral Neuroscience, University of Cincinnati Academic Health Center, PO Box 670559, Cincinnati, OH 452670559, USA. Tel.: +1 513558 4218; Email: robert.krikorian@uc.edu. tance and early type 2 diabetes exhibit diminished cerebral glucose utilization in regions vulnerable to $\mathrm{AD}$ [2]. In addition, insulin receptor resistance and related hyperinsulinemia have been associated with mitochondrial dysfunction [3] as well as with acceleration of beta amyloid deposition [4] and tau hyperphosphorylation [5], characteristic neuropathological features of Alzheimer's disease (AD). Cerebral glucose hypometabolism is prominent in neurodegenerative disorders including AD [6, 7] and Parkinson's disease [8], and has been observed as an early indicator 
of neuronal dysfunction in at-risk individuals well in advance of clinically evident cognitive disturbance [9].

Late-life, sporadic AD develops over a preclinical period of many years during which neuropathological changes accumulate before the appearance of clinical impairment [10]. In higher risk individuals, cerebral glucose hypometabolism is evident during the extended pre-dementia period before other early pathological signs [9]. In Mild Cognitive Impairment (MCI), a condition marked by mild memory decline and increased risk for $\mathrm{AD}$, the proportion of dead to dysfunctional neurons is lower than in $\mathrm{AD}$ [11], suggesting the possibility that progression of neurodegeneration could be slowed if dysfunctional neurons could be rescued.

Ketosis has been shown to produce benefits with respect to metabolic function and fatty acid metabolism in humans [12] and neuroprotection in cellular and animal studies [13-15]. Reduced B-amyloid [16] as well as increased mitochondrial uncoupling protein and decreased reactive oxygen species in the hippocampus [17] have been demonstrated after administration of the ketogenic diet for six weeks and 12 days, respectively. Genetic and biochemical evidence of increased mitochondrial biogenesis has been demonstrated after three weeks' administration of a ketogenic diet in rodents [18]. There have been few human trials assessing neurocognitive benefit in response to dietary ketosis, which can be achieved through carbohydrate restriction or supplementation with medium chain triglycerides (MCT). Older adults with MCI and AD exhibited increased $\beta$-hydroxybutyrate (BHB) levels and acutely improved cognitive function after consumption of MCT [19]. A larger, multicenter intervention also demonstrated cognitive benefit in $\mathrm{AD}$ with longer-term MCT consumption [20]. In addition, there are indications that ketone bodies enhance human cerebral circulation [21] and may signal neuroprotective effects mediated through inhibition of histone deacetylases [22].

The brain is capable of utilizing ketone bodies as an alternate energy substrate to glucose for up to $60 \%$ of its energy requirement [23]. Cerebral glucose utilization declines approximately $18 \%$ in MCI [24] and 20\% to $25 \%$ in $\mathrm{AD}[25]$ relative to non-pathological aging. However, the ability of the aging and AD brain to utilize ketone bodies for energy substrate is normal or near normal $[25,26]$. It has been estimated that raising ketone body concentration to $0.4 \mathrm{mM}$ to $0.5 \mathrm{mM}$ would provide $5 \%$ to $10 \%$ of total brain energy requirement [23], offsetting a substantial portion of the bioenergetic deficit attributed to glucose hypometabolism in MCI and AD. Accordingly, one might consider ketosis a plausible intervention with the potential to restore neuronal bioenergetic function by providing an alternate energy substrate to compensate for impaired glucose metabolism and by protecting against neuropathological insults such as generation of $\beta$-amyloid and hyperphosphorylated tau [14, 27].

In a prior controlled trial, we demonstrated improved memory function in older adults with MCI following six weeks' carbohydrate restriction designed to induce ketogenesis [28]. In conjunction with elevated ketone body levels, improvements of metabolic function and anthropometric factors also were evident, including correction of peripheral hyperinsulinemia and reductions of serum glucose levels, weight, and waist circumference. It was postulated that enhanced bioenergetic function and neuroprotection associated with ketone metabolism contributed to improved memory function, although data were not obtained to assess these factors in that trial. We have followed that study with a trial designed to examine the effects of dietary ketosis on cerebral metabolites associated with neuronal health and bioenergetic function using proton magnetic resonance spectroscopy $\left({ }^{1} \mathrm{H}\right.$ MRS) in a small sample of MCI participants. ${ }^{1} \mathrm{H}$ MRS allowed us to examine in vivo changes in markers of cerebral bioenergetic function in response to the same six-week ketogenic diet intervention in a noninvasive manner. We performed pre- and postintervention ${ }^{1} \mathrm{H}$ MRS with spectra acquired from the dorsolateral region of the right prefrontal cortex. The metabolites of interest were: myo-inositol, Nacetyl-aspartate, creatine + phosphocreatine, choline, glutamate, and glutamate + glutamine. We present data showing change in metabolites implicating enhanced cerebral bioenergetic function and performance on cognitive tasks.

\section{Material and methods}

\subsection{Participants}

The study protocol was approved by the University of Cincinnati Medical Institutional Review Board, and each enrolled participant signed the informed consent document. Prospective participants were recruited 
from the greater Cincinnati area with print advertising in the form of flyers posted at senior centers and advertisements placed in the Cincinnati Enquirer, the major daily newspaper. The recruitment ads solicited participation of older adults with mild, acquired memory decline for a dietary intervention study. There was no stipulation in the recruitment material as to weight or metabolic status with the exception that those with diabetes were to be excluded. We enrolled seven participants. However, ${ }^{1} \mathrm{H}$ MRS data for two of the participants possessed insufficient signal-to-noise ratio to permit robust quantification. The final sample included five participants (two men, three women). The mean age of the sample was $72( \pm 7.1)$ years, and the mean educational level was $16( \pm 2.0)$ years.

\subsection{Procedure}

Prospective participants were assessed with structured interview instruments to determine eligibility for study inclusion. All reported age-related memory decline such as forgetfulness and prospective memory difficulty and inefficiencies in everyday activities but not substantial functional decline. None of the participants required assistance with everyday functioning that might imply advanced cognitive impairment or dementia. The Academic and Medical History Questionnaire [29] was used to obtain demographic information and information regarding academic attainment, current and past medical conditions, and medication and substance use. Those who reported substance abuse or who had been diagnosed with diabetes or psychiatric or neurological conditions were excluded as well as those treated regularly with medications such as benzodiazepine and stimulant drugs that might affect outcome measures. Level of memory impairment as manifested in everyday activities was determined with the Clinical Dementia Rating (CDR), which elicited information from the participant and an informant (typically, spouse or adult child) concerning the nature and extent of cognitive decline at home and in the community [30]. The functional domains of memory, orientation, problem solving, community affairs, home activities, and personal care were evaluated, and the ratings for each domain contributed to a global CDR classification with the memory domain weighted most heavily. Global CDR classifications included no impairment, mild decline, and mild, moderate, and severe dementia. We enrolled individuals with mild decline (coded 0.5 ), cor- responding to $\mathrm{MCI}$, and excluded those with $\mathrm{CDR}$ classifications indicating no impairment or dementia. In addition to the global CDR classification, we derived the sum of boxes score from the arithmetic sum of the category ratings across the six domains of functioning, which served as a means of quantifying overall level of functional decline [31].

Qualifying participants were administered the brain imaging and neurocognitive protocols at the enrollment visit, which occurred one day before the dietary intervention was initiated, and at the final visit, which was scheduled to occur after the intervention had been performed for six weeks. Anthropometric measures and blood samples also were obtained at the enrollment and final visits.

Brain imaging and spectroscopy measurements were performed at the University of Cincinnati College of Medicine Center for Imaging Research using a 4.0 Tesla Varian Unity INOVA Whole Body MRI/MRS system (Agilent, Palo Alto, CA). All MRI and MRS pulse sequences were controlled by VnmrJ graphic user interface.

A 3-D, whole-head image was acquired using a Modified Driven Equilibrium Fourier Transform (MDEFT, T $\mathrm{MD} 1.1 \mathrm{~s}$, TR $13 \mathrm{~ms}$, TE $6 \mathrm{~ms}$, FOV $25.6 \times 25.6 \times 19.2 \mathrm{~cm}$, flip angle $20^{\circ}$ ) pulse sequence [32] to allow for selection of the MRS voxel based on anatomical structure and for the quantification of tissue content. ${ }^{1} \mathrm{H}$ MRS data were obtained from a $2 \mathrm{~cm} \times 2 \mathrm{~cm} \times 2 \mathrm{~cm}$ voxel placed in the dorsolateral region of the right prefrontal cortex. After MRS voxel positioning, the magnetic field homogeneity was optimized using FASTMAP (Fast Automatic Shimming Technique by Mapping Along Projections) [33]. The localized, single-voxel spectrum was acquired with a PRESS sequence (Point Resolved Spectroscopy) with water suppression using TR $3000 \mathrm{~ms}$, TE $30 \mathrm{~ms}$, 128 averages, $1500 \mathrm{~Hz}$ spectral width, and 1024 data points with water suppression by the VAPOR (variable pulse powers and optimization relaxation delays) method [34]. For the purposes of determining absolute concentrations for metabolites, the water signal from the same voxel served as an internal reference. The unsuppressed water signal spectra were acquired using the same acquisition parameters with the qualification that only four averages and a lower receiver gain were used [35]. Tissue contents (percent gray matter, white matter, and cerebrospinal fluid) of the MRS voxel were obtained through tissue segmentation of the MDEFT images using SPM5 software. 
The ${ }^{1} \mathrm{H}$ MRS spectra were analyzed using LCModel software to quantify the following cerebral metabolite levels: myo-inositol (mInos), a cell membrane component; $\mathrm{N}$-acetyl-aspartate (NAA), a marker of neuronal integrity; creatine + phosphocreatine $(\mathrm{Cr})$, a marker of mitochondrial energy turnover; choline (Ch), representing choline-containing compounds glycerophosphocholine and phosphocholine; glutamate (Glu); and glutamate + glutamine (Glx) [36]. The metabolite levels were further corrected for tissue dependence with tissue segmentation and corrected for the relaxation loss with transverse and longitudinal relaxation times [37, 38]. Metabolite levels are presented in concentration $(\mathrm{mM})$ relative to unsuppressed water signal from the same MRS voxel.

Neurocognitive assessments included measures of working memory, long-term memory, and mood. The Trail-making Test part B [39] was used to assess working memory with respect to ability to switch mental set in accordance with changing task demands [40]. In this timed, paper and pencil procedure, participants were presented with a two dimensional array of randomly arranged alphanumeric characters and asked to alternately sequence digits and letters by drawing connecting lines with a pencil. The outcome measure was time on task so that a lower score reflected better performance. We evaluated secondary or long-term memory ability with the California Verbal Learning Test (CVLT) [41]. This is a list-learning task consisting of presentation of 16 common words over five learning and testing trials. Among a number of learning and retention measures, we used list recall after a delay period as an index of long-term memory. Alternate forms of the CVLT were administered at the enrollment and final visits to eliminate the possibility of retention or priming of specific item content at the second administration. Mood was assessed with the Geriatric Depression Scale (GDS) [42], a 30-item inventory designed to evaluate symptoms of depression in older adults.

We also gathered data on potential mediators of neurocognitive function and the dietary intervention. We measured body weight and waist circumference at the narrowest waist between the lowest rib and iliac crest. Blood samples were obtained after overnight fast for determinations of serum glucose and insulin values by radioimmunoassay and enzymatic assays by the Biochemistry Laboratory of the University of Cincinnati Clinical Translational Research Center. BHB concentration in serum was measured from samples obtained at the enrollment and final visits by enzymatic quantification at the University of Cincinnati Mouse Metabolic Phenotyping Center.

\subsection{Dietary intervention}

The prescribed dietary intervention involved carbohydrate restriction intended to promote ketogenesis. Dietary regimens with $20 \mathrm{~g}$ to $50 \mathrm{~g}$ carbohydrate per day have been shown to produce adaptation to ketone metabolism and are associated with the detectable presence of ketone bodies [43]. Adaptation to ketone metabolism begins after several hours of carbohydrate restriction. Within three to four days, the brain begins to utilize ketone bodies for energy with full adaptation occurring after two to three weeks $[44,45]$. Carbohydrate was the only restricted macronutrient. Consumption of protein and fat was allowed to vary, and total energy intake was not constrained. Very high levels of fat ( $90 \%$ of total kilocalorie intake) have been prescribed traditionally to induce ketosis for seizure management [46]. However, recent trials have indicated that consumption of moderate levels of protein does not suppress ketogenesis [47, 48] or seizure control $[49,50]$, allowing for a less severe regimen.

Participants were given information as to the macronutrient constituents of common foods and counseled as to sources of carbohydrate, fat, and protein. The participants were advised to consume not more than $20 \mathrm{~g}$ carbohydrate per day as a target level in order to help insure adaptation to ketone metabolism. Fruit was prohibited and carbohydrate consumption was limited to moderate portions of vegetables. Specific foods that might be included or eliminated were discussed with each participant in light of information as to food items typically consumed based on pre-intervention diet diaries and consultation with each participant. We maintained weekly contact with participants throughout the intervention to answer questions and promote adherence to the protocol.

Participants also completed daily diet diaries during the entire intervention. This was done not only to provide information concerning food intake but also to increase participant attention to food items consumed and, thereby, promote adherence to the regimen. In addition, diet records were completed the week before the enrollment visit as a record of pre-enrollment consumption habits. Participants received oral and written instruction for recording quantities of all foods 
and beverages consumed and used portion posters (Nutrition Consulting Enterprises, Framingham, MA, USA) to estimate food and beverage portions. The completed diet records were reviewed with each participant to clarify ambiguities related to particular foods and portion sizes. Three-day records were extracted for analysis from the seven-day periods before the enrollment and final visits to derive pre- and postintervention data concerning change in energy and macronutrient intake. These records were selected based on temporal proximity to the study visits and consisted of diaries for three consecutive days including one weekend day. Food records were analyzed for kilocalorie and macronutrient content by the Bionutrition Core of the Cincinnati Children's Hospital Medical Center Clinical Translational Research Center using the Nutrition Data Systems for Research (NDSR) software (Nutrition Coordinating Center, University of Minnesota, Minneapolis, MN, USA).

\section{Results}

As noted, all participants were classified with Mild Cognitive Impairment on the basis of a CDR global score of 0.5 . The mean $(S D)$ CDR sum of boxes score was $0.8(0.44)$, consistent with this level of memory decline. Table 1 contains information obtained prior to and during the final week of the interven-

\section{Table 1}

Anthropometric, metabolic, macronutrient, and serum BHB information at enrollment and after six weeks' carbohydrate restriction

\begin{tabular}{lccc}
\hline & $\begin{array}{c}\text { Pre- } \\
\text { intervention }\end{array}$ & Final & $t ; p$ \\
\hline Body weight, kg & $81.9(20.6)$ & $75.7(18.8)$ & $7.85 ; 0.0001^{*}$ \\
Waist circumference, cm & $92.7(19.4)$ & $89.0(20.0)$ & $6.29 ; 0.003^{*}$ \\
Fasting glucose, mg/dL & $108.7(22.7)$ & $104.3(13.4)$ & $0.98 ; 0.37$ \\
Fasting insulin, $\mu \mathrm{U} / \mathrm{mL}$ & $23.0(10.7)$ & $20.5(5.8)$ & $1.07 ; 0.34$ \\
Total energy, kcal & $1684(453)$ & $950(138)$ & $4.32 ; 0.01^{*}$ \\
Carbohydrate, g & $203(80)$ & $40(22)$ & $4.18 ; 0.01^{*}$ \\
Protein, g & $68(16)$ & $64(18)$ & $0.88 ; 0.42$ \\
Animal, g & $40(14)$ & $58(22)$ & $2.18 ; 0.04^{*}$ \\
Non-animal, g & $27(5)$ & $6(4)$ & $6.59 ; 0.002^{*}$ \\
Fat, g & $67(26)$ & $60(11)$ & $0.46 ; 0.66$ \\
Fiber, g & $24(5)$ & $11(8)$ & $3.71 ; 0.02^{*}$ \\
B-hydroxybutyrate, mM & $0.09(0.10)$ & $0.49(0.3)$ & $3.09 ; 0.03^{*}$ \\
\hline
\end{tabular}

Note. All values are mean (SD). Statistical analyses are dependent sample $t$-tests. ${ }^{*}$ indicates significant effect at $p \leq 0.05$. Daily energy and macronutrient intake were calculated from three-day diet diaries generated during the week before enrollment and during the final week of the intervention. tion concerning anthropometric variables, metabolic factors, total daily energy and macronutrient consumption, and ketone body concentration. At enrollment, anthropometric and metabolic measures were typical of US adults aged 60 years and older with respect to abdominal obesity, hyperinsulinemia, and elevated fasting glucose $[51,52]$. The pre-intervention mean serum $\mathrm{BHB}$ concentration was at the level expected after overnight fast [24].

Dependent sample $t$-tests were performed to assess change in the outcome measures. At the end of the intervention the mean $\mathrm{BHB}$ concentration was significantly increased $(p=0.03)$, and elevations relative to pre-intervention were apparent for each participant with values ranging from $+0.21 \mathrm{mM}$ to $+0.90 \mathrm{mM}$ indicating that the level of carbohydrate restriction maintained by the participants was adequate to increase ketone body concentrations. As shown in Table 1, we observed statistically significant anthropometric and dietary changes across the six-week intervention including reductions of body weight $(-7.5 \%)$, waist circumference $(-3.9 \%)$, daily energy intake $(-43 \%)$, carbohydrate intake $(-80 \%)$, and fiber intake $(-54 \%)$. Notably, overall protein and fat consumption did not change appreciably with the intervention. With the decline in carbohydrate intake, the amount of animal protein increased and non-animal protein intake decreased. Glucose and insulin values also declined, although not significantly.

After six weeks' carbohydrate restriction there was a significant increase in mInos $(p=0.02)$ and trends for increased NAA $(p=0.09)$ and $\mathrm{Cr}(p=0.11)$. There was no change in Ch $(p=0.36)$, Glu $(p=0.51)$, and Glx $(p=0.23)$. Figure 1 shows change from the enrollment to final visit in mInos, NAA, and $\mathrm{Cr}$ values for each participant. All of the participants demonstrated increases in mInos and four the five participants showed increases in NAA and Cr. Notably, the fifth participant showed the smallest increase in mInos and no change in NAA and Cr.

We also observed cognitive performance changes at the end of the six week intervention. Dependent $t$-test analyses indicated that executive ability, as measured by task switching efficiency on the Trail-making part B, was significantly improved $(t=4.39, p=0.01)$. In addition, there was a trend for improved performance on the CVLT list recall task $(t=2.44, p=0.07)$. There was no change in level of mood symptoms $(t=0.53, p=0.62)$.

We examined the relationship of change in the anthropometric, dietary, and metabolic factors with 

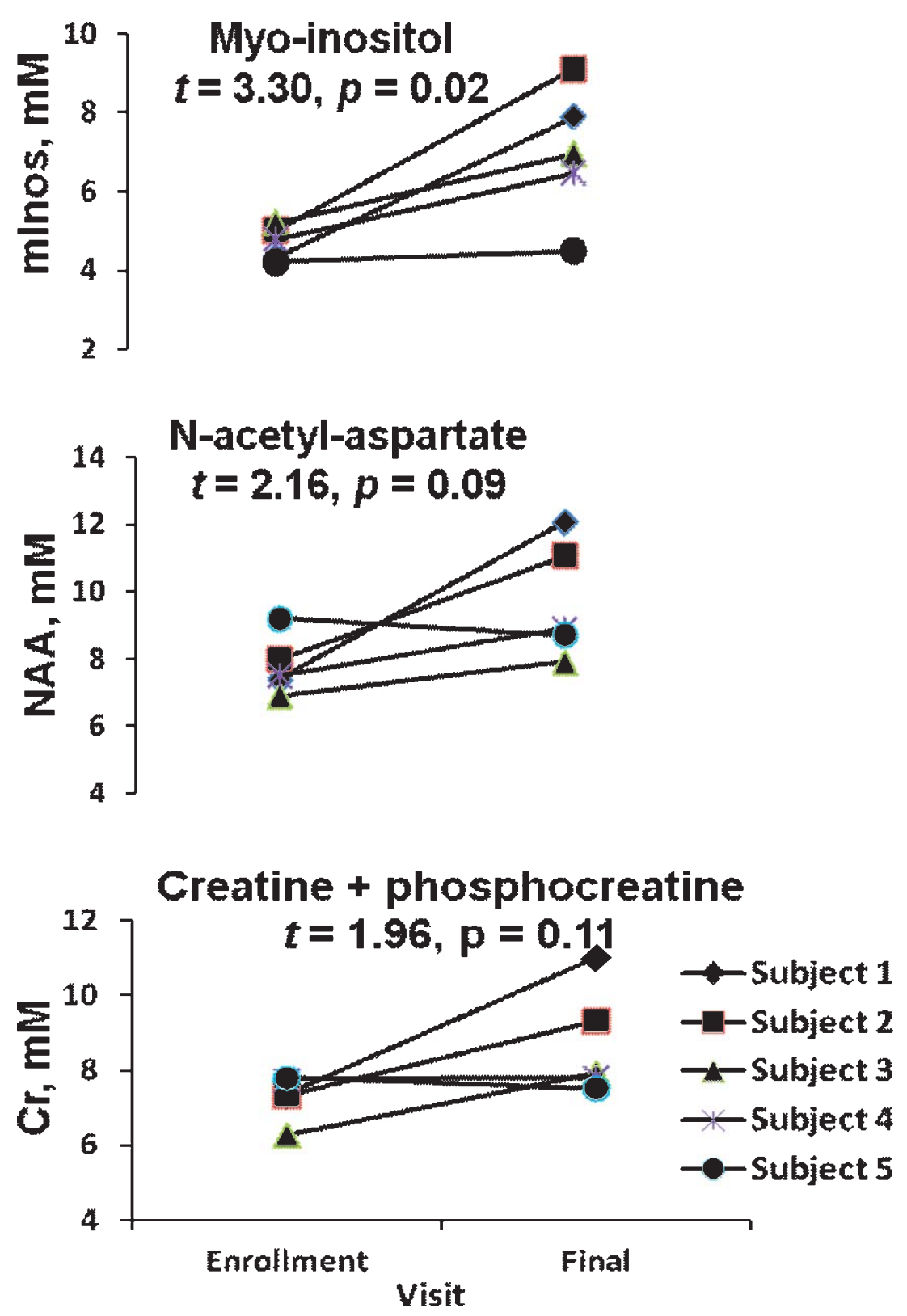

Fig. 1. Change from enrollment to final study visits in proton MRS metabolites, myo-inositol (mInos), N-acetyl-aspartate (NAA), and creatine + phosphocreatine $(\mathrm{Cr})$, for each participant. Participants 1 through 4 showed increases in all metabolites at the final visit. Statistical tests are dependent sample $t$-tests and indicate a significant effect for mInos and trends for NAA and Cr.

change in working memory and long-term memory performance using regression analyses. In these analyses, the dependent variable was the final visit cognitive performance score (either Trail-making or CVLT recall) and the predictor variables were the corresponding enrollment visit cognitive score and the biological or dietary factor change score (final visit less enroll- ment visit). These analyses were intended to isolate the association of change in the biological or dietary factor with the increment in cognitive performance. Body weight, waist circumference, and total daily energy intake decreased significantly, but were not associated with the cognitive improvements. Change in body weight was not related to Trail-making (standardized 
$\left.\beta\left(\beta_{S t d}\right)=-0.05, p=0.84\right)$ or list recall $\left(\beta_{S t d}=-0.02\right.$, $p=0.90)$ performance. Change in waist circumference was not related to Trail-making $\left(\beta_{S t d}=0.04\right.$, $p=0.88)$ or list recall $\left(\beta_{\mathrm{Std}}=0.04, p=0.74\right)$ performance. Change in total energy intake was not related to Trail-making $\left(\beta_{S t d}=0.10, p=0.69\right)$ or list recall $\left(\beta_{S t d}=0.33, p=0.37\right)$ performance. However, change in serum $B \mathrm{HB}$ was associated inversely with Trailmaking time on task $\left(\beta_{S t d}=-0.38, p=0.05\right)$ but not list recall $\left(\beta_{S t d}=0.10, p=0.36\right)$.

As is evident in Fig. 1 the increases in the brain metabolites mInos, NAA, and $\mathrm{Cr}$ were smaller or absent for participants 4 and 5. We examined demographic, anthropometric, metabolic, and cognitive factors in an effort to identify differences in any of these variables that might be related to the relatively weaker neurochemical response in these two participants. The only other factor that was different for participants 4 and 5 was the change in $B \mathrm{HB}$. For each these participants, the increase in $\mathrm{BHB}$ concentration was higher ( $0.81 \mathrm{mM}$ and $0.59 \mathrm{mM}$, respectively), whereas the difference for the other three subjects was in the range of $0.2 \mathrm{mM}$.

\section{Discussion}

To our knowledge, this is the first study to investigate the effect of dietary ketosis on cerebral metabolites in individuals with MCI. Despite the small sample size, we observed marginal trends for increases in the cerebral metabolites NAA and $\mathrm{Cr}$, which are associated with neuronal integrity and cellular energy turnover. These observations are contrary to what has been reported in non-interventional and cross-sectional MRS studies of AD and MCI in which decreased levels of NAA and $\mathrm{Cr}$ are viewed as markers of neurodegeneration [53]. Accordingly, while the increments in these two metabolites were marginal, they suggest the possibility that neuronal function may have been improved. Further investigation with larger MCI samples is necessary to corroborate and extend these observations and substantiate the notion that moderate-term ketogenesis is associated with improved neuronal integrity and enhanced bioenergetic function in this population.

In addition, there was a significant effect for mInos with increases for all participants. In some but not all observational studies, increased mInos has been considered representative of pathology in MCI and AD and associated with impaired cognitive function. How- ever, a meta-analysis examining magnetic resonance spectroscopic imaging findings in MCI noted that alterations in mInos were not uniform across brain regions and that effect sizes were small relative to alterations in NAA [54]. A number of longitudinal studies have reported no change in mInos in MCI subjects [53, 55-57] or found mInos increased specifically in the context of decreased $\mathrm{Cr}$ [58]. This raises doubt about whether increased mInos can be considered a common or central finding in MCI, especially in the absence of reduced NAA, which has been determined to be a reliable marker of neuronal integrity [54]. The ${ }^{1} \mathrm{H}$ MRS signal arises from free mInos and is reflective of either membrane substrate or membrane breakdown product [59]. Decreased NAA and Cr concentrations in MCI have been thought to indicate neuronal death and impairment of mitochondrial function [54, 58]. However, in the context of stable or increased markers of energy substrate (Cr) and neuronal viability (NAA) and improved cognitive performance, our finding of increased mInos likely does not reflect cell membrane breakdown. Also, the dietary regimen involved, de facto, a decrease in mInos intake because of reduced vegetable consumption and elimination of fruit [60] so that the observed increase in mInos occurred in spite of reduced dietary intake. Again, the increments in NAA, $\mathrm{Cr}$, and in cognitive performance suggest neuronal viability while in dietary ketosis and it is unlikely that the measured increase in mInos was the result neuronal degradation.

Despite the small sample size we found positive changes in cognitive performances on the executive set-shifting and list recall tasks improved. The level of depressive symptoms endorsed by the participants in this sample was quite low and well below the threshold for clinically significant mood disturbance [42].

From the point of view of metabolic function and dietary habits, our participants were similar to those enrolled in other trials involving older adults with MCI [61, 62]. Our recruitment efforts were aimed at enrolling individuals with mild memory decline and, with the exception of diagnosed diabetes, we did not consider metabolic status as an inclusion or exclusion factor. The pre-enrollment anthropometric and metabolic values were consistent with those observed in metabolic syndrome [51, 52], and insulin resistance and hyperinsulinemia characteristic of that syndrome have been implicated in cerebral glucose hypometabolism [2, 3, 7] as well as increased risk for neurodegeneration $[4,63]$. The reductions of 
weight and waist circumference observed at the end of the intervention suggest metabolic benefit, although changes in glucose and insulin were not statistically significant, possibly because of the high variability of values in the small sample. We did not find statistical support for the notion that improving insulin sensitivity was beneficial with respect to neurocognitive function, although it will be of interest to assess the potential benefit of metabolic factors in a larger sample.

We observed a significant decrease in total daily energy intake that almost certainly contributed to the reductions of body weight and waist circumference. The fact that protein and fat consumption did not change appreciably with the intervention indicates that essentially all of the reduction in energy intake was attributable to the reduction in carbohydrate consumption. This recapitulates the dietary behavior observed in the prior trial [28], in which the low carbohydrate group exhibited reductions in carbohydrate and total calorie intake but no change in protein and fat consumption. Also, as in that prior trial, we found no relationship between change in weight or change in calorie intake and cognitive performance.

With regard to ketogenesis, elevation of BHB concentration was evident for each of the participants. The individual values and the significant elevation of mean $\mathrm{BHB}$ concentration at the end of the intervention were in the expected range, consistent with mild dietary ketosis, and well below the concentration indicative of pathological ketoacidosis [64]. These data provide evidence that the level of carbohydrate restriction was adequate to stimulate mild ketosis, and the post-intervention $\mathrm{BHB}$ concentration was in the range considered sufficient to provide cerebral bioenergetic recovery [23]. Although change in BHB level did not predict the cerebral metabolite levels, it was associated with performance on the Trail-making task (but not list recall). It is possible that a longer intervention period or stricter regimen might have generated higher serum $\beta$ HB levels, greater improvements, and stronger associations of cognitive performance with BHB levels. On the other hand, one must assume that there is individual variation in ketone body synthesis and utilization, which might contribute to the differential cerebral metabolite responses. While the ability of the aging and $\mathrm{AD}$ brain to utilize ketone bodies for energy substrate is not diminished [25] it is likely that individual variability in this metabolic adaptation affected the range of final $\mathrm{BHB}$ values.
The major limitations of this study are the small sample size and uncontrolled design, and the study results must be judged to be preliminary. However, we did observe positive increments in the primary outcome measures, and the findings are consistent with other evidence supporting the notion that mild dietary ketosis is associated with bioenergetic enhancement. One would expect stronger effects with a larger sample, and it will be of interest to determine the extent to which neurocognitive enhancements associated with the ketogenic diet intervention are related to enhanced bioenergetic function in distinction from other putative benefits [13, 16, 17, 22, 65]. On balance, these findings are novel and provocative and consistent with our prior research [28] and with empirically-derived expectations concerning the neurobiological benefits associated with ketone metabolism.

\section{Acknowledgments}

Elizabeth M Fugate contributed essential assistance with MRS data acquisition. This study was supported by an anonymous gift for Alzheimer's disease prevention research.

\section{References}

[1] Ford ES, Giles WH, Dietz WH. Prevalence of metabolic syndrome among US adults. JAMA. 2002;287:356-9.

[2] Baker LD, Cross DJ, Minoshima S, Belongia D, Watson GS, Craft S. Insulin resistance and Alzheimer-like reductions in regional cerebral glucose metabolism for cognitive normal adults with prediabetes and early type 2 diabetes. Arch Neurol. 2011:68:51-7.

[3] Henderson ST. High carbohydrate diets and Alzheimer's disease. Med Hypotheses. 2004;62:689-700.

[4] Craft S. Insulin resistance syndrome and Alzheimer's disease: Age- and obesity-related effect on memory, amyloid, and inflammation. Neurobiol Aging. 2005;26:S65-9.

[5] Freunde S, Plum L, Schnitker J, Leeser U, Udelhoven M, Krone W, Bruning JC, Schubert M. Peripheral Hyperinsulinemia Promotes Tau Phosphorylation In Vivo. Diabetes. 2005;54:3343-8.

[6] Costantini LC, Barr LJ, Vogel JL, Henderson ST. Hypometabolism as a therapeutic target in Alzheimer's disease. BMC Neurosci. 2008;9:S16.

[7] Reiman EM, Chen K, Alexander GE, Caselli RJ, Bandy D, Osborne D, Saunders AM, Hardy J. Correlations between apolipoprotein $\mathrm{E} \varepsilon 4$ gene dose and brain-imaging measurements of regional hypometabolism. PNAS. 2005;102:8299302.

[8] Bohnen NI, Koeppe RA, Minoshima S, Giordani B, Albin RL, Frey KA, Kuhl KE. Cerebral Glucose Metabolic Features of 
Parkinson Disease and Incident Dementia: Longitudinal Study. J Nucl Med. 2011;52:848-55.

[9] Reiman EM, Chen K, Alexander GE, Caselli RJ, Bandy D, Osborne D, Saunders AM, Hardy J. Functional brain abnormalities in young adults at genetic risk for late-onset Alzheimer's dementia. PNAS. 2004;101:284-9.

[10] Sperling RA, Aisen PS, Beckett LA, Bennett DA, Craft S, Fagan AM, Iwatsubo T, Jack CR Jr, Kaye J, Montine TJ, Park DC, Reiman EM, Rowe CC, Siemers E,Stern Y, Yaffe K, Carrillo MC, Thies B, Morrison-Bogorad M, Wagster MV, Phelps $\mathrm{CH}$. Toward defining the preclinical stages of Alzheimer's disease: Recommendations from the National Institute on Aging-Alzheimer's Association workgroups on diagnostic guidelines for Alzheimer's disease. Alzheimer's Dement. 2011;7:280-92.

[11] Morrison JH, Hof PR. Life and death of neurons in the aging brain. Science. 1997;278:412-9.

[12] Volek JS, Fernandez ML, Feinman RD, Phinney SD. Dietary carbohydrate restriction induces a unique metabolic state positively affecting atherogenic dsylipidemia, fatty acid partitioning, and metabolic syndrome. Prog Lipid Res. 2008:47: 307-18.

[13] Gasior M, Rogawski MA, Hartman AL. Neuroprotective and disease-modifying effects of the ketogenic diet. Behav Pharmacol. 2006;17:431-9.

[14] Kashiwaya Y, Takeshima T, Mori N, Nakashima K, Clarke K, Veech RL. D- $\beta$-hydroxybutyrate protects neurons in models of Alzheimer's and Parkinson's disease. Proc Natl Acad Sci. 2000;97:5440-4.

[15] Maalouf M, Rho JM, Mattson MP. The neuroprotective properties of calorie restriction, the ketogenic diet, and ketone bodies. Brain Res Rev. 2009;59:293-315.

[16] van der Auwera I, Wera S, Van Leuven F, Henderson ST. A ketogenic diet reduces amyloid beta 40 and 42 in a mouse model of Alzheimer's disease. Nutri Metabol. 2005;2:28.

[17] Sullivan PG, Rippy NA, Dorenbos K, Concepcion RC, Agarwal AK, Rho JM, The ketogenic diet increases mitochondrial uncoupling protein levels and activity. Ann Neurol 2004;55:576-80

[18] Bough KJ, Wetherington J, Hassel B, Pare JF, Gawryluk JW, Greene JG, Shaw R, Smith Y, Geiger JD,Dingledine JD. Mitochondrial biogenesis in the anticonvulsant mechanism of the ketogenic diet. Ann Neurol. 2006;60:223-35.

[19] Reger MA, Henderson ST, Hale C, Cholerton B, Baker LD, Watson GS, Hyde K, Chapman D, Craft S. Effects of Bhydroxybutyrate on cognition in memory-impaired adults. Neurobiol Aging. 2004;25:311-4.

[20] Henderson ST, Vogel JL, Barr LJ, Garvin F, Jones JJ, Constantini LC. Study of the ketogenic agent AC-1202 in mild to moderate Alzheimer's disease: A randomized, doubleblind, placebo-controlled, multicenter trial. Nutri Metabol. 2009;6:31.

[21] Hasselbalch SG, Madsen PL, Hageman LP, Olsen KS, Justesen N, Holm S, Paulson OB Changes in cerebral blood flow and carbohydrate metabolism during acute hyperketonemia. Am J Physiol. 1996;270:E746-51.

[22] Newman JC, Verdin E. Ketone bodies as signaling metabolites. Trends Endocrin Metab. 2013.

[23] Cunnane S, Nugent S, Roy M, Courchesne-Loyer A, Croteau E, Tremblay S, Castellano A, Pifferi F, Bocti C, Paquet N,
Begdouri H, Bentourkia M, Turcotte E,Allard M, BarbergerGateau P, Fulop T, Rapoport SI. Brain fuel metabolism, aging, and Alzheimer's disease. Nutrition. 2011;27:3-20, doi:10.1016/j.nut.2010.07.021.

[24] Mosconi L, Tsui WH, De Santi S, Li J, Rusinek J, Convit A, Li Y, Boppana M, de Leon MJ. Reduced hippocampal metabolism in MCI and AD: Automated FDG-PET image analysis. Neurology. 2005:64:1860-7.

[25] Lying-Tunell U. Lindblad BS, Malmlund HO, Persson B. Cerebral blood flow and metabolic rate of exygen, glucose, lactate, pyruvate, ketone bodies and amino acids. Acta Neurol Scan. 1981;63:337-50.

[26] Nugent S, Tremblay S, Chen KW, Ayutyanont N, Roontiva A, Castellan C, Fortier M, Roy M, Courchesne-Loyer A, Bocti C, Lepage M, Turcotte E, Fulop T, Reiman EM, Cunnane SC. Brain glucose and acetoacetate metabolism: A comparison of young and older adults. Neurobiol Aging. 2014;35: 1386-95.

[27] Kashiwaya Y, Bergman C, Lee J, Wan R, King MT, Mughal MR, Okun E, Clarke K, Mattson MP, Veech RL. A ketone ester diet exhibits anxiolytic and cognition-sparing properties, and lessens amyloid and tau pathologies in a mouse model of Alzheimer's disease. Neurobiol Aging. 2013;34: 1530-9.

[28] Krikorian R, Shidler MD, Dangelo K, Couch SC, Benoit SC, Clegg DJ. Dietary ketosis enhances memory in mild cognitive impairment. Neurobiol Aging. 2012;33:425.e19-425.e27.

[29] Busch RM, Farrell K, Lisdahl-Medina K, Krikorian R. Corsi block-tapping task performance as a function of path configuration. Journal of Clinical and Experimental Neuropsychology. 2005;27:127-34.

[30] Hughes CP, Berg L, Danziger WL, Coben LA, Martin RL. A new clinical scale for the staging of dementia. Brit J Psychia. 1982:140:566-72.

[31] Lynch CA, Walsh C, Blanco A, Moran M, Coen RF, Walsh JB, Lawlor BA. The clinical dementia rating sum of box score in mild dementia. Dement Geriatr Cogn. 2006;21:40-3.

[32] Lee J-H, Garwood M, Menon R, Adriany G, Andersen P, Truwit $\mathrm{CL}$, Ugurbil K. High contrast and fast three-dimensional magnetic resonance imaging at high fields. Magn Reson Med. 1995;34:308-12.

[33] Gruetter R, Boesch C. Fast, noniterative shimming of spatially localized signals. In vivo analysis of the magnetic field along axes. J Magn Reson. 1992;96:323-34.

[34] Tkac I, Staruck Z, Choi IY, Gruetter R. In vivo ${ }^{1} \mathrm{H}$ NMR spectroscopy of rat brain at $1 \mathrm{~ms}$ echo time. Magn Reson Med. 1999;41:649-56.

[35] Kreis R, Ernst T, Ross BD. Absolute quantitation of water and metabolites in the human brain. II. Metabolite concentrations. J Magn Reson B. 1993;102:9-19.

[36] Provencher SW. Estimation of metabolite concentration from localized in vivo proton NMR spectra. Magn Reson Med. 1993;30:672-79.

[37] Ernst T, Kreis R, Ross B. Absolute quantification of water and metabolites in human brain. I. Compartments and Water $\mathbf{J}$ Magn Reson B. 1993;102:1-8.

[38] Hetherington H, Mason G, Pan J, Ponder S, Vaughan J, Tweig D, Pohost GM. Evaluation of cerebral gray and white matter metabolite differences by spectroscopic imaging at 4.1T. Magn Reson Med. 1994;32:565-71. 
[39] Reitan RM. Trail Making Test: Manual for administration and scoring. Tucson, AZ: Reitan Neuropsychology Laboratory; 1992.

[40] Sanchez-Cubillo I, Perianez JA, Adrover-Roig D, RodriguezSanchez JM, Rios-Lago M, Tirapu J, Barcelo F. Construct validity of the Trail Making Test: Role of task-switching, working memory, inhibition/interference control, and visuomotor abilities. J Int Neuropsycholo Soc. 2009;15:438-50.

[41] Delis DC, Kramer JH, Kaplan E, Ober BA. California Verbal Learning Test, 2nd ed. San Antonio, TX: Psychological Corporation. 2000.

[42] Yesavage JA, Brink TL, Rose TL. Development and validation of a geriatric depression rating scale: A preliminary report. J Psychiatr Res. 1983;7:37-49.

[43] Westman EC, Feinman RD, Mavropoulos JC, Vernon MC, Volek JS, Wortman JA, Yancy WS, Phinney SD. Lowcarbohydrate nutrition and metabolism. Am J Clin Nutri. 2007; 86:276-84.

[44] Cahill G. Starvation in man. New Engl J Med. 1970;282:66875.

[45] Phinney SD, Bistrian Br, Wolfe RR, Blackburn GL. The human metabolic response to chronic ketosis without calorie restriction. Metabolism. 1983;32:757-68.

[46] Vining EP, Freeman JM, Ballaban-Gil K, Camfield CS, Camfield PR, Holmes GL. A multicenter study of the efficacy of the ketogenic diet. Arch Neurol. 1998;55:1433-7.

[47] Boden G, Sargrad K, Homko C, Mozzoli M, Stein TP. Effect of a low-carbohydrate diet on appetite, blood glucoselevels, and insulin resistance in obese patients with Type 2 Diabetes. Ann Intern Med. 2005;142:403-11.

[48] Cassady BA, Charboneau NL, Brys EE, Crouse KA, Beitz DC, Wilson T. Effects of low carbohydrate diets high in red meats or poultry, fish, and shellfish on plasma lipids and weight loss. Nutr Met. 2007;4:23.

[49] Kossoff EH, Dorward JL. The modified Atkins diet. Epilepsia. 2008;49S:37-41.

[50] Kosoff EH, Krauss GL, McGrogan JR, Freeman JM. Efficacy of the Atkins diet as therapy for intractable epilepsy. Neurology. 2003;61:1789-91.

[51] Ervin R. Prevalence of metabolic syndrome among adults 20 years of age and over by sex, age, race and ethnicity and body mass index. National Health Statistics Reports, no. 13. Hyattsville, MD: National Center for Health Statistics. 2009.

[52] Grundy SM, Cleeman JJ, Daniels SR, Donato KA, Eckel RH, Franklin BA, Gordon DJ, Krauss RM, Savage PJ, Smith SC, Spertus JA, Costa F. Diagnosis and management of the metabolic syndrome: An American Heart Association/National Heart, Lung, and Blood Institute scientific statement. Circulation. 2005;112:2735-52.
[53] Foy CM, Daly, EM, Glover A, O’Gorman R, Simmons A, Murphy DG, Lovestone S. Hippocampal proton MR spectroscopy in early Alzheimer's disease and mild cognitive impairment. Brain Topography. 2011:24:314-22.

[54] Tumati S, Martens S, Aleman A. Magnetic resonance spectroscopy in mild cognitive impairment: Systematic review and meta-analysis. Neurosci Biobehav Rev. 2013; doi: 10.1016/j.neubiorev.2013.08.004.

[55] Catani M, Cherubini A, Howard R, Tarducci R, Pelliccioli GP, Piccirilli M, Gobbi G, Senin U, Mecocci P. ${ }^{1} \mathrm{H}$ MR spectroscopy differentiates mild cognitive impairment from normal brain aging. Neuroreport. 2001;12:2315-7.

[56] Kantarci K, Weigand SD, Jack CR. Longitudinal ${ }^{1} \mathrm{H}$ MRS changes in mild cognitive impairment and Alzheimer's disease. Neurobiol Aging. 2007;28:1330-9.

[57] Modrego PJ, Fayed N. Longitudinal magnetic resonance spectroscopy as marker of cognitive deterioration in mild cognitive impairment. Am J Alzheimer's Dis Other Demen. 2012;26:631-6.

[58] Yang ZX, Huo SS, Cheng XF, Xu ZF, Coa Z, Zeng JX, Xiao YY, You KZ, Chen W, Lui YY, Wu RH. Quantitative multivoxel proton MR spectroscopy study of brain metabolites in patients with amnestic mild cognitive impairment: A pilot study. Neuroradiol. 2012;54:451-8.

[59] Stork C, Renshaw PF. Mitochondrial dysfunction in bipolar disorder: Evidence from magnetic resonance spectroscopy research. Mol Psychia. 2005;10:900-19.

[60] Clements RS, Darnell B. Myo-inositol content of common foods: Development of a high-myo-inositol diet. Am J Clin Nutr. 1980;33:1954-67.

[61] Krikorian R, Shidler MD, Nash TA, Kalt W, Vinqvist-Tymchuk MR, Shukitt-Hale B, Joseph JA. Blueberry supplementation improves memory in older adults. J Agr Food Chem. 2010;58:3996-4000.

[62] Krikorian R, Boespflug EL, Fleck DE, Stein AL, Wightman JD, Shidler MD, Sadat-Hossieny S. Concord grape juice supplementation and neurocognitive function in human aging. $\mathrm{J}$ Agri Food Chem. 2012;60:5736-42.

[63] Luchsinger JA, Tang MX, Shea S, Mayeux R. Hyperinsulinemia and risk of Alzheimer disease. Neurol. 2004;63:1187-92.

[64] Courchesne-Loyer A, Fortier M, Tremblay-Mercier J, Chouinard-Watkins R, Roy M, Nugent S, Castellano CA, Cunnane SC. Stimulation of mild, sustained ketonemia by medium-chain triacylglycerols in healthy humans: Estimated potential contribution to brain energy metabolism. Nutrition. 2013; 4:635-40.

[65] Veech RL, Chance B, Kashiwaya Y, Lardy HA, Cahill GF. Ketone bodies, potential therapeutic uses. IUBMB Life. 2001;51:241-7. 